\title{
Generation and development of surface waves on the interface boundary of viscous fluid oscillating drop
}

\author{
Leonid Tonkov and Alena Chernova* \\ Institute of Mechanics UB RAS, 426067, 34 T. Baramzinoy Str., Izhevsk, Russia
}

\begin{abstract}
Numerical experimental methods for surface waves' generation and development processes associated with oscillations of small liquid droplet on a solid surface vibrating at a frequency close to $1 \mathrm{kHz}$ are used. Internal flows formed inside the droplet are characterized by vivid threedimensionality, complex topology, are determined by the contact angle and surface waves propagation. Interaction processes are analysed. The achieved results are compared with the experimental ones.
\end{abstract}

\section{Introduction}

When small liquid droplet oscillates in heave we can observe surface ripple waves excitement. Its dynamics depends on viscosity, surface tension and liquid density as well as external action characteristics. Let's consider preliminary stage of the phenomenon with small amplitudes that endure liquid heave oscillations on its surface to initiate capillary gravity waves. The present thesis deals with a numerical research on internal flows in a small amount of liquid (a droplet) placed on a low vibrating solid surface and its influence on the generation and development of surface waves.

We can define some articles [1-3] in experimental hydrodynamics that study oscillations of liquid with ultra-small volume $(5 \mu \mathrm{l})$ placed on a solid substrate vibrating at low frequencies (up to $800 \mathrm{~Hz}$ ) and with low amplitudes (up to $22 \mu \mathrm{m}$ ). Data on droplet free surface depending on the vibrating surface location are provided, and droplet shapes and internal capillary flows corresponding to various surface vibration modes are also considered.

Current analytical works are dedicated to the droplet free surface profile location matters $[4,5]$ and the dynamic process is described only in works $[6,7]$. Thus only work [7] studies problems concerning surface waves distribution on a free surface of an ideal liquid droplet under axisymmetric statement.

Current numerical works studying the droplet dynamics on vibrating surfaces are very few $[8,10]$. The work [10] numerically studies the oscillation and spraying process of a single liquid droplet from the solid rod. In this study the physical problem statement has several rather strong assumptions: the initial droplet configuration was defined as half sphere, in addition to this the possible movement of a contact line was not considered; in

*Corresponding author: alicaaa@gmail.com 
hydrodynamic equations, cycling acceleration of a substrate is added to the gravity acceleration. Also the work [10] describes only solutions for the two dimensional problem.

Experimental research proves that internal flows in a droplet are characterized as vividly 3 dimensional. The volume of fluid (VOF) method was applied in this work.

\section{Problem statement}

We consider the problem of liquid droplet motion caused by vertical movements of a solid substrate. Let the area $\Omega \in R^{3}$ be filled with biphasic medium and $\Omega=\Omega_{1} \Omega_{2}$ with subarea $\Omega_{2}$ containing a gas, and $\Omega_{1}$ containing a liquid; $\Gamma_{0}$ is the phase boundary, and $\Gamma_{1}$ is the atmospheric boundary of computational region. At the initial instant $t=0$ the substrate starts to oscillate in heave at a given frequency $(\mathrm{f}=635 \mathrm{~Hz})$ and amplitude $(\mathrm{a}=3.5 \mu \mathrm{m})$ corresponding to the 8th mode [2] of shape oscillation. A liquid drop placed on the substrate also starts to oscillate in heave.

The experimental apparatus and procedure is described in article [2]. Water droplet of $5 \mu \mathrm{l}$ volume was placed in the substrate center (fig. 1), thus the wetting angle was equal to $\Theta=115 \pm 1^{\circ}$, and the contact diameter and droplet height, $2.02 \mathrm{~mm}$ and $1.52 \mathrm{~mm}$, respectively.

\section{Methods and algorithms}

The VOF method [11] describes the droplet dynamics as a system of two none-mixable uncompressible viscous fluids; the motion of each is described by Navier-Stokes equations, a continuity equation and additional nonstationary volume fraction transport equation [12]. The study [14] shows that free surface dynamics requires accounting for the wetting angle dynamic change in a substrate/liquid/gas triple point for adequate solution. The contact angle description will be performed according to the model

$$
\Theta=\Theta_{0}+\left(\Theta_{A}-\Theta_{R}\right) \operatorname{th}\left(\frac{u_{W}}{u_{\Theta}}\right),
$$

thereby in initial conservation equation system, written according to the VOF method, only yhr motion equation will be changed

$$
\frac{\partial \rho U}{\partial t}+\nabla \cdot(\rho U U)=-\nabla p+\nabla \cdot \tau-\sigma K \nabla \alpha \delta(\alpha)(1-\cos \Theta)+\rho g,
$$

where $K$ - surface curvature $\Gamma_{0}, \sigma$ - surface tension coefficient, $\Theta$ - wetting angle, $\alpha-$ scalar-valued function [12], aiming as liquid volume fraction.

Boundary conditions are defined in a following way: - on a border $\Gamma_{1}: \partial u / \partial n=0, p=p_{0}$, where $n$ - outward normal; - on liquid/substrate border $\partial p / \partial n=0, u_{x}=u_{z}=0$, $u_{y}=u_{\Omega 0}$ where $\mathrm{u}_{\Omega 0}-$ velocity of substrate motion.

Studies $[13,14]$ describe the detailed process of this equation system solution. The cartesian grid consists of 364,140 hexagons and has axial and radial refinement. Studis [13, 14] study the liquid droplet oscillation process on a substrate vibrating at low frequencies corresponding to low (second and fourth) oscillation modes. Let's consider that solution of the liquid oscillation at frequency corresponding to high (6 and 8) oscillation modes require correction of $\Theta_{R}$ and $\Theta_{A}$, in respect to similar constants determined for low modes of liquid oscillations. 


\section{Numerical results}

This study investigates problem on oscillation at frequency $635 \mathrm{~Hz}$ of a water drop $5 \mu \mathrm{l}$ in volume on a solid substrate, that is experimentally described [6]. Mathematical simulation of liquid motion under oscillations was performed using volume-of-fluid method in a cell in two stages: first - the solution of liquid droplet stationary problem [14]; second - the liquid droplet dynamics simulation with vibrating substrate acting on it.

Calculated droplet vertical cross-section profiles (fig. 1, a) fit the experimental ones (fig.1, b) - 4 circle brake lines of free surface were detected [2] that proves the mathematical model and results of computational procedure.
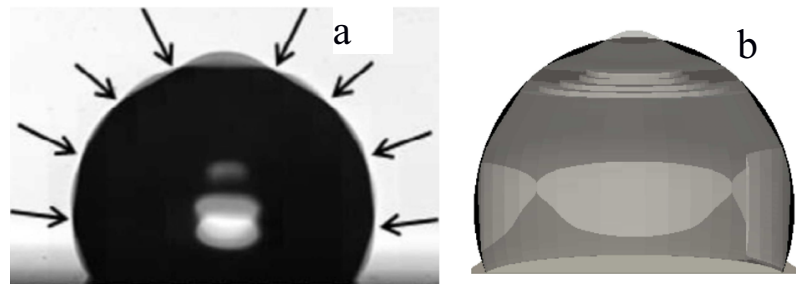

Fig. 1. Form of a droplet obtained in the result of: a) experiment [2]; b) present calculation

Fig. 2 describes the velocity field and free surface deformation. Fig. 2 shows that interaction of the diverse flows leads to complex topological as well as whirl structures generation in a droplet. From the point of free surface the described process is followed by generation and development (motion) of surface waves that are localized by topological peculiarities of liquid flow. We need to point out that direction of velocity vectors in a liquid droplet is significantly determined by the substrate motion [14].

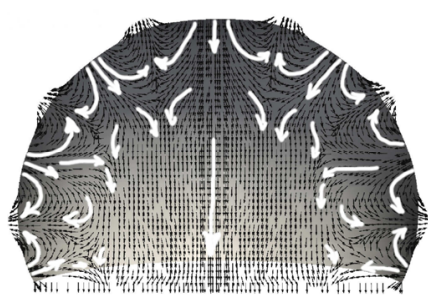

$7.25 t$

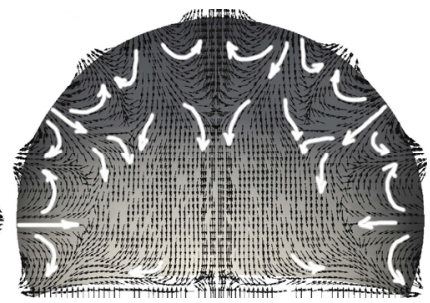

$7.75 t$

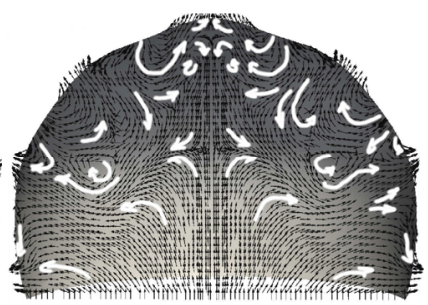

$8 t$

Fig. 2. Internal droplet flows and free surface deformation, $t=\mathrm{t} / T$

Thus, we can suggest that surface waves' generation and development is connected with local topological peculiarities of internal flows, their transformation and mutual interaction.

This work was partially supported by RFBR according to the research project №16-38-00127-mol_a and 16-41-180276r_a.

\section{References}

1. Y.S. Shin, H.Ch. Lim, Eur. Phys. J.E. 37, 1 (2014)

2. H. Kim, H.Ch. Lim, J. Phys. Chem. B. 119, 6740 (2015) 
3. Ch.S. Park, H. Kim, H.Ch. Lim, Exp. Thermal Fluid Sci. 78, 112 (2016)

4. V.Z. Kanchukaev, Pisma v ZhPh. 30(2), 12 (2004)

5. D.V. Lyubimov, T.P. Lyubimova, S.V. Shklyaev, Fluid Dynamics 39, 851 (2004)

6. A.O. Ivancov, Bulletin of Perm University. Series: Physics. 3, 16 (2012)

7. D.V. Lyubimov, T.P. Lyubimova, S.V. Shklyaev, Phys. Fluids 18, 012101 (2006)

8. Y. Li, A. Umemura, Int. J. Multiphase Flow 60, 64 (2014)

9. G.B. Foote, J. Comp. Phys. 11, 507 (1973)

10. A.J. James, M. Smith, A. Glezer J. Fluid Mech. 476, 29 (2003)

11. V.A. Kalinichenko, S.V. Nesterov, A.N. So, Fluid Dynamics 50, 535 (2015)

12. C.W. Hirt, B.D. Nichols, J. Comp. Phys. 39, 201 (1981)

13. A.A. Chernova, S.P. Kopysov, L.E. Tonkov, IOP Conf. Ser.: Mater. Sci. Eng. 158, 012026 (2016)

14. I.M. Kuzmin, A.S. Sarmakeeva, A.A. Chernova, Chemical Physics and Mesoscopy. 18, $515(2016)$ 